\title{
Ergodicity in Economics: a Decision Theoretic Evaluation
}

\author{
Luciano Andreozzi*
}

June 15, 2021

\begin{abstract}
Peters (2019) discusses a compound lottery that allegedly reveals a weakness of the way uncertainty is modeled within economics. This note evaluates that example in the light of standard expected utility. I obtain two results. First, using a variant of Rabin (2000) calibration theorem, I show that the paradox Peters obtains only arises because he implicitly assumes an unbounded utility function for money. That when utility is unbounded such paradoxical outcomes can arise is a well-known fact in decision theory, so his contribution on this matter is of limited interest. Second, I use the same example to illustrate Peters' claim that, when applied to compound lotteries, expected utility is based on the implicit assumption that the underlying stochastic process is ergodic. I compare Peters' analysis with the original one due to Samuelson (1971) and show that this claim is unfounded.

Keywords Expected utility - Independence Axiom - St. Petersburg paradox - Risk Aversion - Bounded utility - Ergodicity economics

JEL Classification C73
\end{abstract}

\section{Introduction}

In a recent article, Peters (2019) makes several claims about the way in which decisions under uncertainty are modelled within economic theory. Peters has a generally very low opinion about the economists' way of modelling uncertainty. He believes that "Expected utility theory is an 18th-century patch, applied to a flawed conceptual framework established in the 17th century that made blatantly wrong predictions of human behavior." Peters \& Adamou (2018)

The author presents his contribution as a first step towards the construction of a more rigorous approach he dubs ergodicity economics, in which uncertainty and time will be dealt with at the same time. The new approach will solve

*luciano.andreozzi@unitn.it. Universitá degli Studi di Trento. Aknowledgements. The present version of this paper benefited from an e.mail exchange with Peter Wakker. 
some long-standing puzzles that are left unsolved by expected utility theory (EUT). Among them, the St. Petersburg paradox, the equity premium puzzle, the evolutionary advantage of cooperation and the rise of inequality.

So far the reactions from economists have been negative Doctor et al. (2020). This note contributes to this debate by assessing a specific part of this program, the one that deals exclusively with the way in which uncertainty is treated in economics. Peters's treatment of this issue revolves around the stochastic process generated by a compound gamble that was originally introduced by Paul Samuelson (Samuelson, 1971). Peters makes two related claims. First, he pretends to show that EUT makes blatantly absurd predictions when dealing with this specific compound lottery. Second, he maintains that the root of this problem lies in the way in which EUT deals with compound lotteries. In his view, hidden in the formalism of EUT there is the implicit assumption that the stochastic process generated by such lotteries is ergodic. Failing this assumption, absurd consequences ensue.

I shall argue that Peters' contribution does not make a significant progress with respect to the existing knowledge the topics he addresses. To show this, in Section 2 I will first deliver a basic reformulation of his running example in terms of standard EUT. Since ergodicity economics has attracted the attention of many scholars who are not acquainted with EUT, my presentation will be intentionally pedagogical. This section delivers nothing more than an adaptation of a well known argument developed by Samuelson (1963). In Section 3 I will prove a very simple proposition that shows that the new paradox Peters proposes requires the utility function for money to be unbounded. In this sense, his example is just another version of the St. Petersburg paradox, and can be solved in the same way.

Section 4 deals with the claim that, in dealing with compound lotteries, EUT implicitly assumes that the corresponding stochastic process is ergodic. I will show this claim to be incorrect, although for a rather subtle reason. In fact, even P. Samuelson's original contribution on this matter is not entirely satisfying. Finally, Section 5 argues that so far the contribution of ergodicity economics to our understanding of decision under uncertainty has been limited, although it may open some interesting lines of research on non-expected utility.

\section{Compound lotteries}

It is not easy to evaluate Peter's claims about EUT, because little effort is made in his papers to link the criticism that he levels against it to the axiomatic approach that became common after von Neumann and Morgernstern's work (von Neumann \& Morgenstern, 1947, Savage, 1954).

In Peters \& Gell-Mann (2016) we read that "Prominent texts in decision theory make incorrect statements about the equality of expectation values and time averages". The only reference the authors give in which this type of error is made is the following passage taken from Chernoff \& Moses (2012):

If a game is favorable from the point of view of the expectation 
value and you have the choice of repeating it many times, then it is wise to do so. For eventually, your amount of money and, consequently, your utility are bound to increase (assuming that utility increases if money increases) (p. 98).

I will rephrase this prescription in the following informal criterion:

Samuelson's collegue's criterion Suppose that, given an initial wealth level $w$ a Decision Maker (DM) prefers lottery $L$ to a sum of money $S$. Then if she is asked $n$ times to choose between $L$ and $S$ she should always choose $L$ over $S$.

The reason for our terminology is Paul Samuelson's famous example involving a colleague of his, who would refuse an equal chance of losing $\$ 100$ or winning $\$ 200$, but would accept a sufficiently large repetitions of the same lottery (Samuelson, 1963). The intuition behind this criterion is that $n$ repetitions of the same lottery cannot be worst, and may be better, than a single one. Its appeal stems from the (incorrect) belief that repeating a lottery many times reduces risk.

In the passage we quoted above, Peters apparently says that Samuelson's colleague criterion is somewhat implied by EUT. His criticism consists in revealing the apparent absurd conclusions that derive from this criterion. Curiously enough, to illustrate his point he uses an example originally proposed by Samuelson himself (Samuelson, 1971). The example runs as follows. The DM, endowed with an initial wealth $w$, receives a sum of money $S_{0}$. She is given the opportunity to participate to a lottery in which a coin is flipped repeatedly and, at each repetition, on Head the DM wins $50 \%$ of her current winning while on Tail she loses 40\% 1 After $n$ rounds the game stops and the DM gets whatever sum of money she has at that time. Notice that at each coin flip the expected monetary value of the lottery increases. At first, one may conclude that, if given the opportunity, a DM may prefer to re-flip the coin after every round, provided she is not too risk averse. The gist of Peters's argument consists in showing that in accepting $n$ repetitions of this lottery, for $n$ sufficiently large the DM will in fact lose money almost surely. The reason behind this paradox is that the stochastic process generated by the repeated coin flip is not ergodic, and hence the evolution over time of the DM's winnings is not properly represented by its expected value.

Figure 1 illustrates this point. It represents (on logarithmic scale) several independent trials of the way the winnings of the DM who starts with $\$ 100$ evolve as the number of rounds $n$ goes from 1 to 500 . Contrary to what one would expect, while the expected monetary value of the lottery increases linearly with $n$ (orange straight line), in most of the trials the DM's winning will steadily decays to zero. The evolution of winnings over time is better described by the geometric mean (blue line). The rising expected monetary value is produced by a small number of outliers with astronomically large winnings.

\footnotetext{
${ }^{1}$ In Samuelson's original example each dollar invested in the lottery produced 2.75 dollars on Head and 0.3 dollars on Tail.
} 


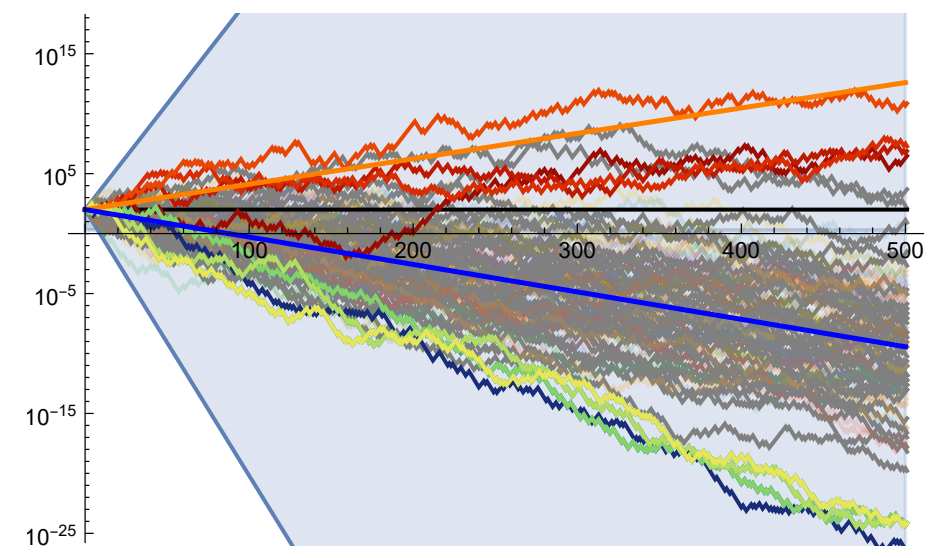

Figure 1: A decision maker starts with $100 \$$ and at every round either wins .5 of looses .4 of what she earned in the previous rounds. The graph plots on logarithmic scale the DM winnings as the number of coin flips increases from 1 to 500. The expected monetary value is described by the orange straight line. However, for most of the realizations the DM will lose money. The graph represents the five most successful runs (in red hues) the least successful runs (in blue hues) and a sample of other 10.000 runs (in light gray).

To assess what believers in EUT can learn from this example, we shall make it slightly more rigorous. For every sum of money $S$, we shall denote with $\delta_{S}$ the degenerate lottery that gives the DM her initial wealth $w$ plus $S$. Consider now the following choice problem:

Example 2.1. The DM has a fixed wealth level $w$ and is given the opportunity to choose among

1. A sum of money $S_{0}$ for sure, that is $\delta_{S_{0}}$;

2. A lottery $L_{S_{0}}^{1}$ that pays $S_{0} \times 1.5$ with probability $\frac{1}{2}$ and $S_{0} \times 0.6$ with probability $\frac{1}{2}$;

3. A lottery $L_{S_{0}}^{n}$ in which the DM starts with $S_{0}$ and at each of $n>1$ rounds the money at round $r-1$ is multiplied with equal probability by 1.5 or 0.6 . The payoff of the lottery is the money the DM has at the last round.

Figure 2 (a) depicts lottery $L_{100}^{2}$. The DM starts with $\$ 100$ and a coin is flipped twice. The red node is reached after two heads and the winning is thus $\$ 100 \times 1.5 \times 1.5=\$ 225$. The lottery $\bar{L}_{100}^{2}$ (panel b) is obtained from $L_{100}^{2}$ by replacing the red node with a lottery in which the winning at that node $(\$ 225)$ is multiplied by 1.5 and 0.6 with the same probability. EUT is based on the independence axiom, that is on the assumption that $\bar{L}_{100}^{2}$ is preferred to lottery $L_{100}^{2}$ only if the DM accepts such a replacement, that is if she prefers a coin 

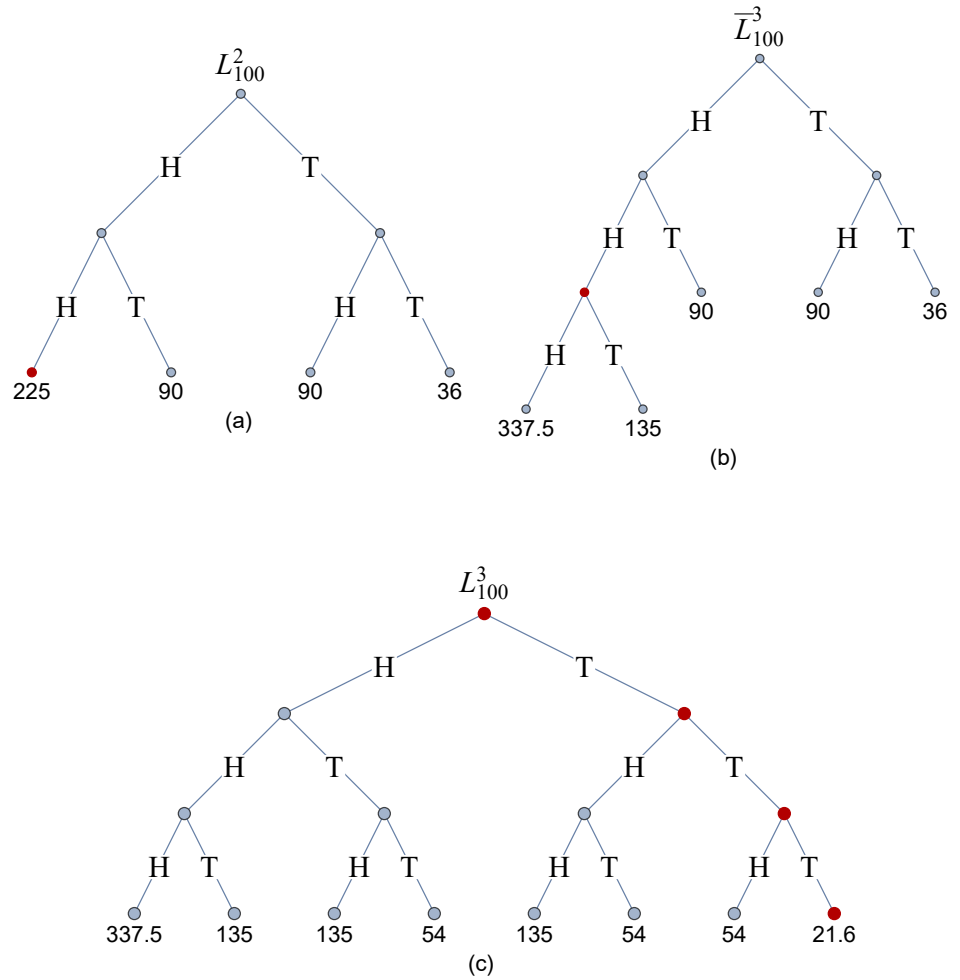

Figure 2: Panel $(a)$ : A coin is tossed twice. At every Head the winning is multiplied by $1+\frac{5}{10}$ at every Tail by $1-\frac{4}{10}$. The initial stake is $100 \$$. Panel $(b)$ : The same lottery, but the red node has been replaced with a further coin toss. Panel $(c)$ : All terminal nodes have been replaced.

flip that yields either $\$ 337.5$ or $\$ 135$ to $\$ 225$ for sure. In our notation, it must be the case that $L_{225}^{1}$ is better than $\$ 225$ for sure. Lottery $L_{100}^{3}$ (panel c) is obtained from $\bar{L}_{100}^{2}$ by replacing the remaining three terminal nodes in a similar manner. It follows from the independence axiom that for $L_{100}^{3}$ to be preferred to $L_{100}^{2}$ the DM must accept all these replacements.

Peters' argument is based on the incorrect belief that, accepting EUT, one is forced to claim that if $L_{100}^{1} \succ \delta_{100}$ then, for every $n, L_{100}^{n+1} \succ L_{100}^{n}$. Our discussion so far shows that for this to be the case the following, much stronger, assumption needs to be made.

Assumption 2.1. There exist a wealth level $w$ such that for every $S>0$ $L_{S}^{1} \succ \delta_{S}$. 
This assumption makes it transparent what is at the hearth of Peters' proposed paradox. It requires that (at least for one wealth level $w$ ) the DM is willing to trade any sum $S$ that could be added to her wealth $w$ with a lottery that pays with equal probability either $1.5 \times S$ or $0.6 \times S$. For, if this is the

case, one can always create a longer lottery $L_{S_{0}}^{n+1}$ from $L_{S_{0}}^{n}$ by replacing one of the terminal winnings $S$ of the first lottery with a lottery that pays $1.5 \times S$ and $0.6 \times S$. Every time such a replacement takes place, the DM obtains a new lottery that has a slightly larger expected monetary value and a larger risk. Not surprisingly, in the limit as the number of replacements approaches infinity, the lottery comes to have an infinite expected monetary value and an infinite risk. We can summarize what we said so far with the following:

Proposition 2.1. For every $n \geq 1, L_{S_{0}}^{n+1} \succ L_{S_{0}}^{n}$ if and only if Assumption 2.1 holds.

\section{Unbounded utility}

The result in the previous section suggests that the paradox lies in the fact that the DM is allowed to have an unbounded utility for money. As the probability of obtaining astronomically large sums of money goes to zero, the utility from these sums must approach infinity. To make this intuition rigorous one needs a definition of an unbounded utility for money. Samuelson (1977) provides such a definition that only requires hypothetical choices among simple lotteries involving three finite amounts of money. His definition is as follows:

Definition 3.1. We say that the DM utility for money is unbounded if for every pair of monetary amounts $\underline{S}>\bar{S}$ and for every probability $\epsilon>0$ there exist a $S^{*}>\bar{S}$ such that

$$
(1-\epsilon) \delta_{\underline{S}}+\epsilon \delta_{S^{*}} \succ \delta_{\bar{S}}
$$

To appreciate this definition consider the following thought experiment. The DM, with wealth level $w$, can obtain Jeff Bezos' wealth $\bar{S} \gg w$ for sure or a lottery that pays a larger sum $S^{*}>\bar{S}$ with probability $\epsilon>0$ and nothing $(\underline{S}=0)$ with probability $(1-\epsilon)$. The DM utility for money is unbounded if, no matter how small $\epsilon$ is, there is always a sum $S^{*}$ larger than Bezos' wealth such that he will prefer the lottery.

Two remarks are in order. First, as Samuelson makes it clear, whether a DM has an unbounded utility for money is an empirical question, although it is arguably a difficult one to test. Second, we are not requiring that the DM's preferences over lotteries obey the axioms of EUT. In fact, for the definition above to work, the DM's preferences over lotteries may not even be representable by a numerical utility function.

We now turn to a DM whose preferences over lotteries do obey EUT. Hence, a utility function $U(x)$ can be defined so that, given two lotteries $p$ and $q$, if $p$ is preferred to $q$ then the expected utility of $p$ is larger than the expected utility of 
$q$. Our next proposition shows that the paradoxical conclusion that for such a DM it may be the case that $L_{100}^{n+1} \succ L_{100}^{n}$ can only hold true if her utility function for money $U(x)$ is unbounded. This clearly implies that her preferences over money are unbounded as in Definition 3.1. The theorem is close to Rabin (2000). (See Rabin \& Thaler (2001) for a more informal presentation and Bleichrodt et al. (2019) for a recent treatment and a survey of the literature.)

Proposition 3.1. Suppose Assumption 2.1 holds. Then the DM has an unbounded utility for money as defined by Definition 3.1.

Proof. We shall prove our proposition with the help of Figure 3. Let $w$ be the initial wealth and let $S$ be any sum of money. In the graph, on the xaxis we write $S$ rather than $w+S$. Fix a utility level $U(w+S)$ and define $r_{S}:=U(w+1.5 S)-U(w+S)$. Consider now the lottery that pays with equal probability $S \times 1.5 \times 1.5=S \times 2.25$ and $S \times 1.5 \times 0.6=S \times 0.9$. Let $\hat{S}=S \times 1.5 \times \frac{1}{2}(1.5+0.6)$ the expected monetary value of this lottery. We now have to fix a value for $U(w+0.9 S)$ and $U(w+2.25 S)$. Geometrically this amounts to choose two points like $\bar{z}$ and $\underline{z}$ on the two vertical lines corresponding to $0.9 \times S$ and $2.25 \times S$. Convexity requires that both these points lie below the red line. Assumption 2.1 implies that the middle point $\hat{y}$ lies above $y$. The reason is that an equal chance of winning $S \times 2.25$ and $S \times 0.9$ must be better than having $1.5 \times S$. The lowest level of $\bar{z}$ compatible with these assumptions is obtained when $\underline{z}$ is chosen at its highest level, namely on the red line, and $\hat{y}$ is chosen at its lowest level, i.e. horizontally aligned with $y$. So the lowest $\bar{z}$ is obtained where the (green) line that joins these points meets the vertical line passing through $2.25 \times S$. Simple algebra suffices to show that this implies that $U(w+S \times 2.25)-U(w+S \times 1.5) \geq \frac{6}{5} r_{S}$. Consider now any $S^{\prime}=S \times 1.5^{n}$ for some $n>1$. The result above implies that $U\left(w+S \times 1.5^{n}\right) \geq U(w+S)+r_{S}\left(\frac{6}{5}\right)^{n}$. This clearly implies that $U(x)$ is unbounded. It is immediate to check that if the utility function $U(x)$ is unbounded, than the DM utility for money is unbounded as in Definition 3.1 .

Our proposition shows that Peters is wrong in claiming that EUT implies that a DM will always prefer a large number of repetitions of a favorable gamble. Quite the contrary is true. EUT implies that a DM will never accept the gamble $L_{s_{0}}^{n}$ for arbitrarily large $n$, unless her utility for money is unbounded. This is not surprising. Even before EUT was axiomatized, K. Menger proved that a DM with an unbounded utility function for money could be induced to forego any sum of money for sure for the opportunity to play (a version of) the St. Petersburg lottery. (Menger, 1934). The two examples rest on the same logic. If the utility for money is unbounded, no matter how small the probability of winning a lottery is, there is always a prize large enough that makes it more attractive than any sum of money for sure. 


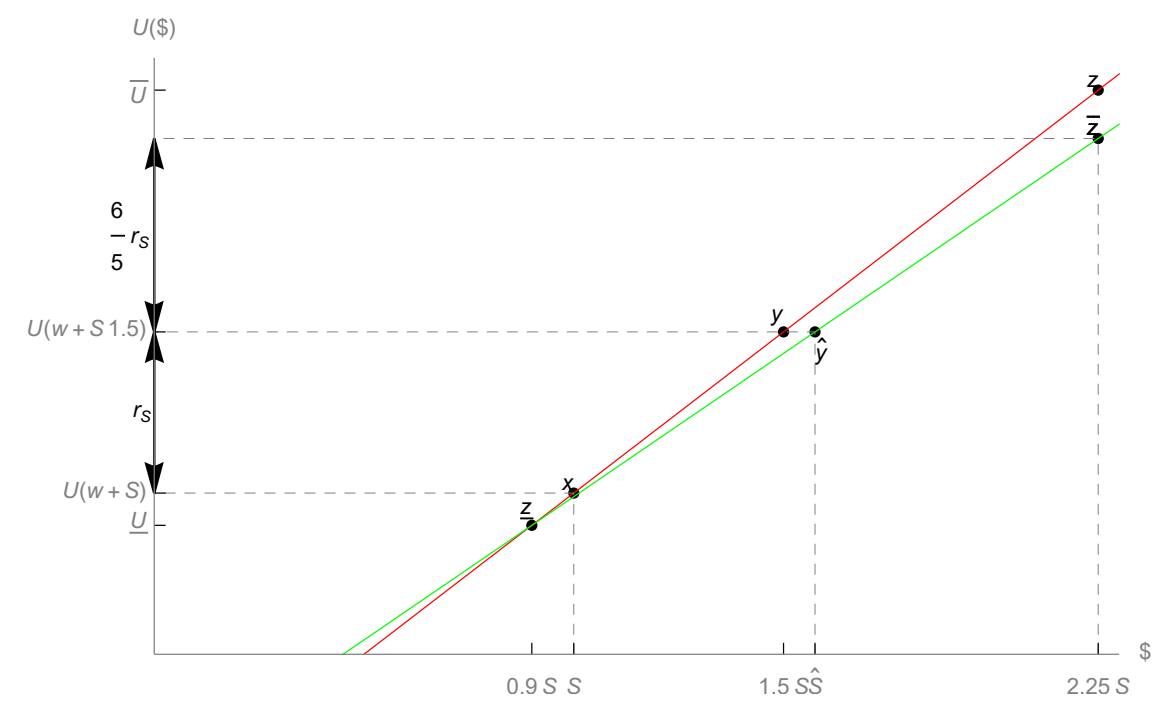

Figure 3: If a DM's preferences over lotteries satisfy Assumption 2.1 then every time a sum of money is added to her wealth her utility grows at least of ??. In turn, this implies that the DM has an unbounded utility for money.

\section{The ergodicity assumption}

We shall now turn to the main criticism that Peters levels against EUT. It is worth quoting it extensively.

my second criticism is more severe and I'm unable to resolve it: in maximizing the expectation value - an ensemble average over all possible outcomes of the gamble - expected utility theory implicitly assumes that individuals can interact with copies of themselves, effectively in parallel universes (the other members of the ensemble). An expectation value of a non-ergodic observable physically corresponds to pooling and sharing among many entities. That may reflect what happens in a specially designed large collective, but it doesnt reflect the situation of an individual decision-maker. Expected utility theory computes what happens to a loosely specified model of my psychology averaged across a multiverse. But I do not live spread out across a multiverse, let alone harvest the average psychological consequences of the actions of my multiverse clones. Ergodicity economics, in contrast, computes what will happen to my physical wealth as time goes by, without appeal to an intangible psychology or a multiverse. We all live through time and suffer the physical consequences (and psychological consequences, for that matter) of the actions of our younger selves.(Peters, 2019, p. 2018) 
To make sense of Peter's argument, consider again $L_{100}^{3}$ in Figure 2. According to EUT, this lottery is better than $\$ 100$ for sure if, given the DM utility function for money $U(x)$ and her wealt $w$ :

$$
\frac{1}{8} U(w+337.5)+\frac{3}{8} U(w+135)+\frac{3}{8} U(w+54)+\frac{1}{8} U(w+21.6)>U(w+100)
$$

Peters' reading of this condition is that EUT evaluates lotteries as the average utility a large population of identical DM would obtain if they were to play this lottery 2 This idea is less unlikely that it looks at first sight. In his original discussion of this example, Samuelson notices that for a risk-neutral individual that he calls Pascal, the inequality above would be true, and it would also be true for any longer lottery $L_{100}^{n}$. Notice that, just like Peters, Samuelson is well aware that "'Almost certainly,' [Pascal] will be 'virtually ruined' in a 'long enough' sequence of play." However, Samuelson insist that

No, Pascal is not a fool according to his criterion. In those rare long sequences (and remember all sequences are finite, albeit very large) when he does experience relatively many wins, he makes more than enough to compensate him [...] for the more frequent times when he is ruined.(Samuelson, 1971, p. 2494)

Apparently, in this passage Samuelson makes just the mistake Peters believes is at the root of EUT: In evaluating the compound lottery $L_{100}^{n}$ for a large $n$, Pascal is averaging out what "he makes" in the vast majority of the cases in which he loses all the money with the much less frequent cases in which he wins astronomically large sums. One may conclude that Peters is right, because Pascal is implicitly assumed to play infinitely many instances of this compound lottery, so that he can "harvest the average psychological consequences of the actions of [his] multiverse clones".

But this is a point on which Samuelson, not EUT, needs a correction. The EUT argument that justifies Pascal's choice is the one we saw in Section 2 . Consider the rightmost final outcome of lottery $L_{100}^{3}$ in Figure 2 (c). This corresponds to Pascal choosing the lottery instead of the $\$ 100$ for sure, flipping the coin three times, loosing at each round and ending up with a meager \$21.6. Note that to get to this outcome Pascal has to pass through the (red) nodes that precede it in the tree. The fact that he is risk-neutral, compounded with the independence axiom, implies that at each of these nodes Pascal prefers to re-flip the coin even if he could stop. This holds true, a fortiori, for all the other terminal nodes, in which his final payoff is larger than $\$ 21.6$. This is the argument that we used in Section 2 to show that $L_{100}^{3}$ is preferred to $\$ 100$ for sure. In no point of this argument we need to assume that the lottery is played more than once or that Pascal is able to reap the benefits he would get in sequences of wins and losses that did not materialize. In fact, it is a

\footnotetext{
${ }^{2}$ Notice that we are using the so-called "terminal wealth" model. Nothing would change if we omitted $w$ using the "income" model. See Cox \& Sadiraj (2006).
} 
straightforward consequence of the independence axiom that for Pascal, as for any other DM how obey EUT, only the current history matters.

\section{Conclusion}

Our main result shows that Peters' claims about what EU prescribes with respect to Example 2.1 are incorrect. A DM who obeys the axioms of EUT will not have a preference for a larger number of coin flips (so it will not be the case that $L_{S_{0}}^{n+1} \succ L_{S_{0}}^{n}$ ) unless her utility function for money is unbounded. This is exactly the condition that Menger (1934) discovered one has to impose to avoid the St. Petersburg paradox. In this sense, the new paradox adds little to what decision theorist already knew on this matter.

Our analysis helps to dispel two other sources of confusion. The first is due to Peters' claim that Menger's result is flawed (Peters, 2011). Peter's argument runs as follows. In the original version of the paradox, the DM is asked how much she would be willing to pay to enter the St. Petersburg lottery. The paradox consists in showing that, if her utility is unbounded, she would be willing to pay an infinite amount of money. Peters believes that Menger's argument is incorrect, because it neglects that, while utility may grow to infinity as the lottery's winnings became increasingly large, it also may go to minus infinity when the price for the ticket goes to infinity and hence the DM wealth (after paying the ticket) becomes negative. Samuelson (1977) had already showed that one can avoid considering these two limits at the same time, by asking what is the sum of money "Santa Claus" should give the DM to convince her not to participate to the St.Petersburg lottery. In this case, the paradox is that, if utility does not have an upper bound, this sum is infinite, which implies that the DM will prefer to receive a ticket for the St. Petersburg lottery rather than Jeff Bezos' wealth for sure. For this argument to work, no sum of money smaller than the DM's original wealth $w$ is involved, so no assumptions have to be made about the DM's utility for arbitrarily small wealth levels. In our treatment of Peters' example, we followed the same route: We fixed the DM initial wealth level and only considered positive increments. This produces a clean result, which only depends on the assumption one makes on amounts of money larger than the DM's initial wealth.

A second source of confusion that hopefully we help to dispel is Peters's claim that EUT is committed to the view that only bounded utility functions are admissible. This is a sensitive topic for ergodicity economics, because the logarithmic utility function is the only admissible in that context.(Peters \& Adamou, 2018). Although this is a position an authority such a K. Arrow has repeatedly held (Arrow, 1974), the prevailing consensus is that there is nothing in EUT that requires utility functions to be bounded and in fact in applications unbounded utility functions are common. A bound on utility function is only required to avoid paradoxical effects in extreme cases like the St. Petersburg lottery, that are of little interest in economic research. As it is often the case, this view has been expressed most clearly by P. Samuelson. In his own words, 
a subject, Paul, whose utility is $\log (x)$

might have to be deemed admissible even if he had to resort to something other than [expected utility] for ordering some of his options. The fact that a thought experiment [like the St. Petersburg lottery] can be contrived does not seem to me to be of great moment: in real life, no Peter or Karl will contrive such an actual experiment to actually confront him with. I agree that the theoretical axiom formulation has a less neat model to handle this postulated Paul: but that is tough doughnuts for the theorist, not for Paul, whose only personal problem is deciding how to choose among options that have no finite expected utilities. (Samuelson, 1977, p. 50)

I close with a final remark for future lines of research. Our analysis hinges crucially on the fact that the DM respects the independence axiom. In Section 4 we showed that this is the key reason why each history of the compound lottery is evaluated in isolation. It is worth exploring to what extent the ergodicity assumption is relevant to those non-expected utility theories that reject the independence axiom. A useful starting point could be (Chew \& Epstein, 1988), that discusses this topic with reference to the Samuelson's colleague criterion. 


\section{References}

Arrow, Kenneth Joseph. 1974. Essays in the Theory of Risk-Bearing. NorthHolland.

Bleichrodt, Han, Doctor, Jason N., Gao, Yu, Li, Chen, Meeker, Daniella, \& Wakker, Peter P. 2019. Resolving Rabin's Paradox. Journal of Risk and Uncertainty, 59(3), 239-260.

Chernoff, H., \& Moses, L.E. 2012. Elementary Decision Theory. Dover Books on Mathematics. Dover Publications.

Chew, S. H., \& Epstein, Larry G. 1988. The Law of Large Numbers and the Attractiveness of Compound Gambles. Journal of Risk and Uncertainty, 1(1), $125-132$.

Cox, James C., \& Sadiraj, Vjollca. 2006. Small- and Large-Stakes Risk Aversion: Implications of Concavity Calibration for Decision Theory. Games and Economic Behavior, 56(1), 45-60.

Doctor, Jason N., Wakker, Peter P., \& Wang, Tong V. 2020. Economists' Views on the Ergodicity Problem. Nature Physics, 16(12), 1168-1168.

Menger, Karl. 1934. Das Unsicherheitsmoment in Der Wertlehre. Zeitschrift fur Nationalokonomie, 5(4), 459-485.

Peters, O., \& Gell-Mann, M. 2016. Evaluating Gambles Using Dynamics. Chaos: An Interdisciplinary Journal of Nonlinear Science, 26(2), 023103.

Peters, Ole. 2011. Menger 1934 Revisited. arXiv:1110.1578 [q-fin], Oct.

Peters, Ole. 2019. The Ergodicity Problem in Economics. Nature Physics, 15(12), 1216-1221.

Peters, Ole, \& Adamou, Alexander. 2018. The Time Interpretation of Expected Utility Theory.

Rabin, M. 2000. Risk Aversion and Expected-Utility Theory: A Calibration Theorem. Econometrica, 68(5), 1281-1292.

Rabin, M., \& Thaler, R.H. 2001. Anomalies: Risk Aversion. The Journal of Economic Perspectives, 15(1), 219-232.

Samuelson, P. 1963. Risk and Uncertainty: A Fallacy of Large Numbers. Scientia, $\mathbf{5 7}(98), 108$.

Samuelson, Paul A. 1971. The "Fallacy" of Maximizing the Geometric Mean in Long Sequences of Investing or Gambling. Proc. Nat. Acad. Sci. USA, 4.

Samuelson, Paul A. 1977. St. Petersburg Paradoxes: Defanged, Dissected, and Historically Described. Journal of Economic Literature, 15(1), 24-55. 
Savage, Leonard J. 1954. The Foundations of Statistics. New York: Wiley.

von Neumann, John, \& Morgenstern, Oskar. 1947. Theory of Games and Economic Behavior. Second edn. New Jersey: Princeton University Press. 